\title{
Interruption Duration
}

National Cancer Institute

\section{Source}

National Cancer Institute. Interruption Duration. NCI Thesaurus. Code C82519.

The time span of a disruption in an event. 